\title{
Improved Pulmonary Inflammation and Fibrosis Progression after Influenza A (H1N1) Infection by Chinese Herbal Medicine: A Case Report
}

\author{
Yao-Zhang Huang, ${ }^{1}$ Mao-Wei Hsieh, ${ }^{2}$ Chien-Hui Hung, ${ }^{3}$ and Chung-Hua Hsu ${ }^{4,5}$ \\ ${ }^{1}$ Department of Radiology, Cheng Hsin General Hospital, Taipei 112, Taiwan \\ ${ }^{2}$ Department of Anesthesiology, Taichung Hospital, Taichung 404, Taiwan \\ ${ }^{3}$ Department of Pediatric, Changhua Christian Hospital, Changhua 500, Taiwan \\ ${ }^{4}$ Institute of Traditional Medicine, National Yang-Ming University, Taipei 112, Taiwan \\ ${ }^{5}$ Branch of Linsen and Chinese Medicine, Taipei City Hospital, Taipei 112, Taiwan \\ Correspondence should be addressed to Chung-Hua Hsu, owlherbs@yahoo.com.tw
}

Received 18 February 2011; Accepted 30 March 2011

Academic Editor: M. Tatar

Copyright ( $) 2011$ Yao-Zhang Huang et al. This is an open access article distributed under the Creative Commons Attribution License, which permits unrestricted use, distribution, and reproduction in any medium, provided the original work is properly cited.

Influenza A (H1N1) virus was the most common cause of human influenza (flu) in 2009. We report a patient infected with influenza A virus who showed improvement in his pulmonary inflammation and fibrosis progression after switching to Chinese herbal medicine (CHM) therapy. Although antiviral drug and corticosteroids were administered, some disturbing respiratory illness and immune imbalance attributed to corticosteroids taken prompted him to seek for CHM therapy. After CHM treatment for three months, significant recovery in chest radiograph was noted, and the result of subsequent pulmonary function test showed improvement.

\section{Introduction}

In the face of the current public health emergency posed by the pandemic of 2009 H1N1 influenza A, many questions arise about whom to treat and how. These questions become even more important in circumstances for which clinical guidance is not available. IDSA, in April of 2009, published clinical practice guidelines focused on the treatment and management of seasonal influenza. In addition, the Center for Disease Control and Prevention (CDC) develops many up-to-date resources to help clinicians address both seasonal influenza and H1N1. However, no one resource can address all questions that arise in day-to-day practice. Many questions continue to be raised about this illness and how to treat it.

To that end, IDSA convened a group of influenza experts to address a number of important questions about the clinical management and antiviral treatment of patients with 2009 H1N1 influenza.

These questions and answers largely reflect expert opinion, as definitive evidence is not yet available. They are not meant to replace the clinical guidance developed by the CDC or IDSA's clinical practice guidelines for seasonal influenza.

The opinions expressed are meant to provide general advice. They are not intended to supplant physician judgment with respect to particular patients or special clinical situations. While IDSA makes every effort to present accurate and reliable information, the information contained in the following questions and answers is "as is" without any warranty of accuracy, reliability, or otherwise, either expressed or implied. Neither IDSA nor its officers, directors, members, employees, or agents will be liable for any loss, damage, or claim with respect to any liabilities, including direct, special, indirect, or consequential damages, incurred in 


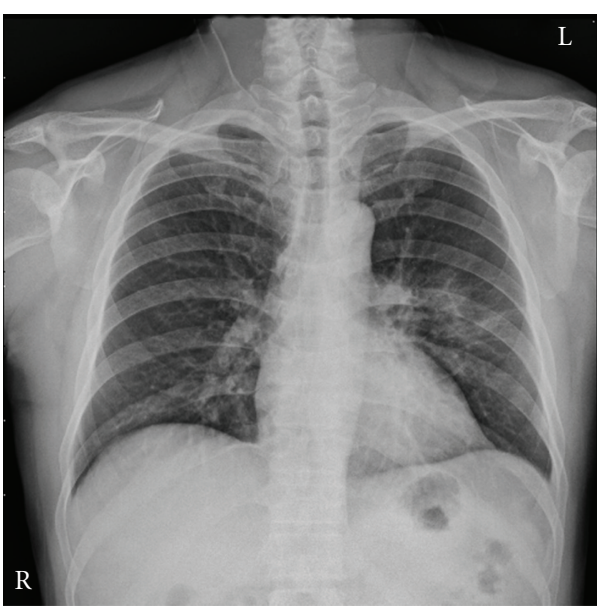

Figure 1: First day after admission-pneumonia over left upper lobe.

connection with these questions and answers or reliance on the information presented.

\section{Case Report}

In late March 2009, an outbreak of a respiratory illness later proved to be caused by novel swine-origin influenza A (H1N1) virus (S-OIV) was identified in Mexico [1]. Subsequent S-OIV cases were observed immediately in many other countries including the United States [2, 3]. The S-OIV patients presented with influenza-like symptoms including fever, headache, myalgia, cough, and running nose. S-OIV infection can cause severe illnesses, severe respiratory diseases such as pneumonia, the acute respiratory distress syndrome, and death in previously healthy persons who are young to middle aged [1]. We report a patient infected with influenza A virus who showed improvement in his pulmonary inflammation and fibrosis progression after switching to Chinese herbal medicine (CHM) therapy.

The patient was a 54-year-old man with a history of hypertension under medical control for two years at the Outpatient Department of Taichung Hospital, Taiwan. He had intermittent cough, and his child had also shown upper respiratory infection symptoms with fever since August 28, 2009. Owing to general malaise, myalgia, and more severe cough with increased amount of dusty, blood tinged sputum, he sought medical help and was then admitted to the hospital on September 1, 2009. Chest radiograph revealed alveolar pattern with air bronchogram over the left upper lobe with lingular segment involvement compatible with pneumonia (Figure 1). After admission, influenza A was found positive after a quick test. He had intermittent shortness of breath, desaturation, and moist over the left lung in chest auscultation on the second day of hospitalization. Progression of pneumonia to the left lung was noticed in the followup chest radiograph (Figure 2). Antiviral drug and antibiotics were given to cover secondary infection and atypical pathogen. Blood culture, sputum culture, and serological testing for organisms causing atypical pneumonia

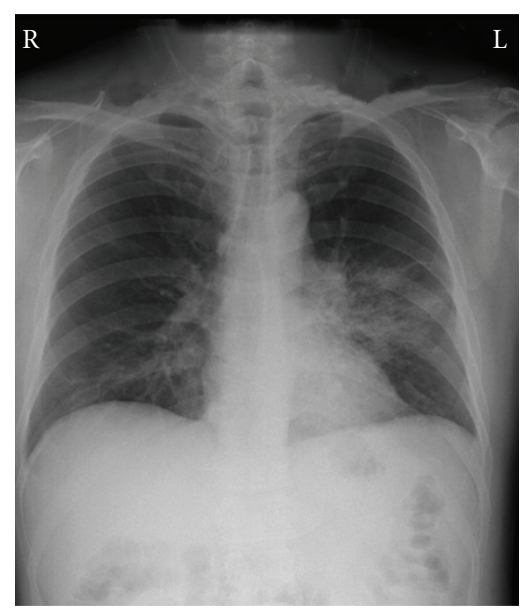

FIGURE 2: Second day of hospitalization-progression of pneumonia to left lung.

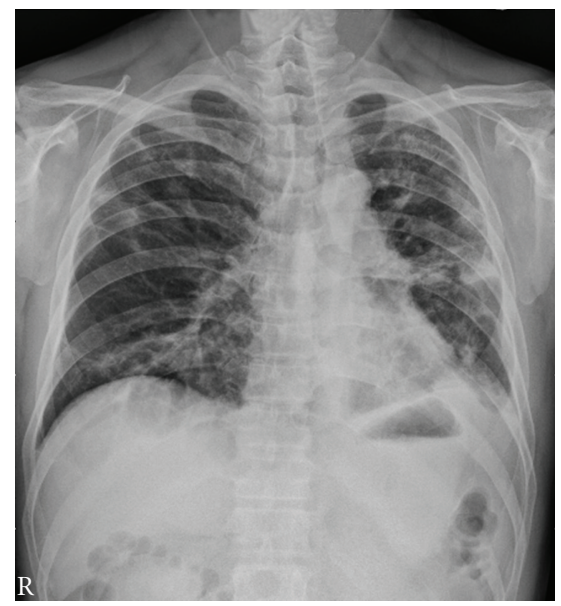

FIGURE 3: First day of CHM therapy-peribronchial infiltration and several consolidative patches at bilateral lung.

were all negative and antibiotics was thus discontinued. However, severe cough with pleuritic pain was noted, and morphine was given for pain control. Chest radiograph showed peribronchial infiltration and several consolidative patches at bilateral lungs (Figure 3 ). The result of pulmonary function test revealed decreased total lung capacity and residual volume (Table 1). He had oxygen dependence, and recovery of his respiratory complications was slow. A large number of corticosteroids and supportive treatments were arranged for pulmonary inflammation and fibrosis progression. However, acne and oral ulcer were noted. Owing to complications of influenza $\mathrm{A}$ infection and the side effects of corticosteroids, he visited our clinic for CHM therapy on October 05, 2009.

According to the traditional Chinese medicine theory, respiratory illness caused by viral infection generally means impairment and abnormal rising of lung Qi accompanied by deficiency of lung Yin. A mixed CHM extract powder that comprised mainly two CHM formulas, Su Zi Jiang 
TABLE 1: Comparison of lung function test results.

\begin{tabular}{lcccc}
\hline & & $2009 / 10 / 08$ & $2009 / 11 / 02$ & $2009 / 12 / 28$ \\
\hline Spirometry & \multicolumn{3}{c}{ BEST (\%PRED) } \\
\hline FVC & Liters & $3.21(94)$ & $3.73(106)$ & $4.78(140)$ \\
FEV1 & Liters & $2.62(94)$ & $2.98(104)$ & $3.97(143)$ \\
FEV1/FVC & $\%$ & 82 & 80 & 83 \\
\hline Lung volumes & & & & \\
TLC & Liters & $3.17(57)$ & $4.11(74)$ & $4.98(90)$ \\
RV & Liters & $-0.04(-2)$ & $0.38(19)$ & $0.03(2)$ \\
\hline
\end{tabular}

FVC: Forced vital capacity.

FEV1: Forced expired volume in 1 second.

TLC: Total lung capacity.

RV: Residual volume.

Qi Tang (Fructus Perillae, Cortex Magnoliae Officinalis, Radix Peucedani, Radix Glycyrrhizae, Rhizoma Pinelliae, Pericarpium Citri Reticulatae, Radix Angelicae Sinensis, Fructus Jujubae, Rhizoma Zingiberis Recens, and Cortex Cinnamomi) and Mai Men Dong Tang (Radix Ophiopogonis, Radix Ginseng, Rhizoma Pinelliae, Radix Glycyrrhizae, Fructus Jujubae, and Semen Oryzae) was given for treatment. The patient was prescribed to take $5 \mathrm{gm}$ of the formula after each meal; that is, 3 times each day making a total daily dose of $15 \mathrm{gm}$. After one month of treatment, there was dramatic improvement in respiratory complications, and both acne and oral ulcer subsided. Therefore, corticosteroids were discontinued. CHM theory states that drugs like steroid and infection due to viruses and other pathogens can damage healthy Qi of the human body. Hence, one of the CHM formulas was changed into Xiang Sha Liu Jun Zi Tang (Radix Aucklandiae, Fructus Amomi, Radix Codonopsis, Rhizoma Atractylodis Macrocephalae, Rhizoma Pinelliae, Poria cocos, Radix Glycyrrhizae, Pericarpium Citri Reticulatae, Rhizoma Zingiberis Recens, and Fructus Jujubae) for regaining health Qi. The total daily dose remained $15 \mathrm{gm}$. After CHM treatment for three months, significant recovery in chest radiograph was noted (Figure 4), and the result of subsequent pulmonary function test showed improvement (Table 1).

\section{Discussion}

Influenza A (H1N1) virus was the most common cause of human influenza (flu) in 2009. On June 11, 2009, the World Health Organization (WHO) raised its pandemic alert level to the highest level, phase 6, indicating widespread community transmission on at least two continents [4]. Case definitions of suspected and confirmed pandemic H1N1 influenza A virus infection are made according to symptoms, signs, and epidemiologic information [5]. Real-time reversetranscriptase(rRT)-PCR or culture is still necessary for the confirmation of pandemic H1N1 influenza A infection. Therefore, we could only suspect that the patient had novel swine-origin influenza A (H1N1) virus infection.

The most common clinical findings of the $2009 \mathrm{H} 1 \mathrm{~N} 1$ influenza A pandemic have been fever, cough, sore throat, malaise, and headache. Vomiting and diarrhea have also

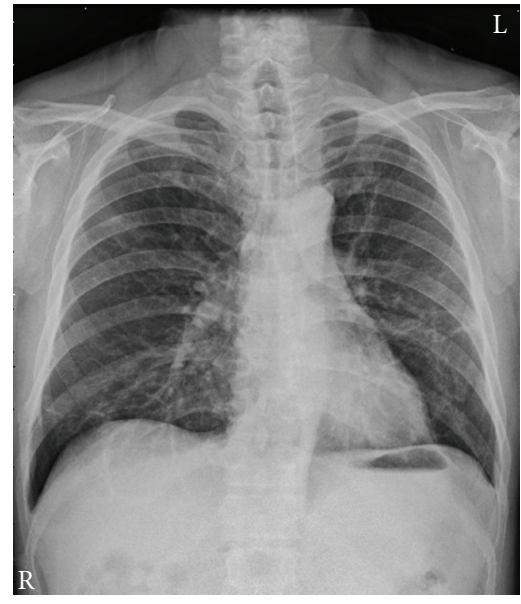

FIgURE 4: Three month after CHM therapy—significant recovery.

been common, both of which are unusual features of seasonal influenza $[6,7]$. Our case had some gastrointestinal symptoms that resemble the clinical presentations of the 2009 pandemic H1N1 influenza A infection.

In pandemic 2009 influenza A (H1N1) infection, bacterial superinfection of the lung has been reported in 4 to 29 percent of cases that resulted in hospitalization or death in the United States [8-12]. Patients with pandemic H1N1 influenza A who develop pneumonia should be treated empirically for community-acquired pneumonia (CAP) [13, 14], given the risk of secondary bacterial pneumonia with organisms such as Streptococcus pneumoniae and Staphylococcus aureus. In our case, the examination results for secondary bacterial pneumonia were all negative, and the prescription of antibiotics was discontinued.

Most cases are characterized by persistent mild illness, and basic supportive care (to relieve aches or fever) is sufficient for most people. Patients who have severe or progressive illness and those with mild symptoms but who are at higher risk for severe illness (e.g., pregnant women, infants and young children, and those with chronic lung problems) should be treated with antiviral medication as soon as possible [15]. In the present case, although antiviral drug and corticosteroids were administered, some disturbing respiratory illness and immune imbalance attributed to corticosteroids taken prompted him to seek for CHM therapy. According to Treatise on Febrile Disease (Shang Han Lun), a variety of herbal formulas have been used to treat patients with infectious diseases for over 1800 years. CHM is generally well accepted among the Chinese population. Prescriptions for infectious diseases were made according to clinical symptoms, rather than laboratory-confirmed pathogens, which were beyond their knowledge at that time. In other words, $\mathrm{CHM}$ formulas treat diseases by regulating the systems of the human body. CHM treatment can reduce the use of steroids to improve inflammation and fibrosis progression and even ameliorate the side effect of steroids. The outbreak of novel influenza and previous SARS posed great challenges. The medical profession had less experience of this unprecedented and deadly disease. Although CHM is 
only a supplementary treatment, these initial findings seem to indicate a possible favorable effect of CHM on SARS or SARS-like diseases in shortening the course of recovery [16]. The experience is worth our further study. Although the findings of the case report need to be verified in a large sample, the initial results showed the benefit of CHM combination therapy for influenza A infection. The definitive benefits need to be further determined.

\section{References}

[1] R. Perez-Padilla, D. De La Rosa-Zamboni, S. Ponce De Leon et al., "Pneumonia and respiratory failure from swine-origin influenza A (H1N1) in Mexico," New England Journal of Medicine, vol. 361, no. 7, pp. 680-689, 2009.

[2] "Outbreak of swine-origin influenza A (H1N1) virus infection-Mexico," Morbidity and Mortality Weekly Report, vol. 58, p. 467, 2009.

[3] World Health Organization, "Influenza-like illness in the United States and Mexico," 2009, http://www.who.int/csr/don/ 2009_04_24/en/index.html.

[4] World Health Organization, "World now at the start of 2009 influenza pandemic," 2009, http://www.who.int/mediacentre/ news/statements/2009/h1n1_pandemic_phase6_ 20090611/en/index.html.

[5] United States Centers for Disease Control and Prevention, "Interim guidance on case definitions to be used for investigations of novel influenza A (H1N1) cases," 2009, http://www. cdc.gov/h1n 1 flu/casedef.htm.

[6] F. S. Dawood, S. Jain, L. Finelli et al., "Emergence of a novel swine-origin influenza A (H1N1) virus in humans," New England Journal of Medicine, vol. 360, no. 25, pp. 2605-2615, 2009.

[7] World Health Organization, "Human infection with new influenza A (H1N1) virus: clinical observations from Mexico and other affected countries," Weekly Epidemiological Record, vol. 84, pp. 185-196, 2009.

[8] J. K. Louie, M. Acosta, K. Winter et al., "Factors associated with death or hospitalization due to pandemic 2009 influenza $\mathrm{A}(\mathrm{H} 1 \mathrm{~N} 1)$ infection in California," Journal of the American Medical Association, vol. 302, no. 17, pp. 1896-1902, 2009.

[9] "Bacterial coinfections in lung tissue specimens from fatal cases of 2009 pandemic influenza A (H1N1)_United States," Morbidity and Mortality Weekly Report, vol. 58, pp. 1-4, 2009.

[10] S. A. R. Webb, V. Pettilä, I. Seppelt et al., "Critical care services and 2009 H1N1 influenza in Australia and New Zealand," New England Journal of Medicine, vol. 361, no. 20, pp. 1925-1934, 2009.

[11] G. Palacios, M. Hornig, D. Cisterna et al., "Streptococcus pneumoniae coinfection is correlated with the severity of H1N1 pandemic influenza," PloS One, vol. 4, no. 12, article e8540, 2009.

[12] R. J. Murray, J. O. Robinson, J. N. White et al., "Communityacquired pneumonia due to pandemic A(H1N1)2009 influenzavirus and methicillin resistant Staphylococcus aureus coinfection," PLoS ONE, vol. 5, no. 1, article e8705, pp. 1-9, 2010.

[13] United States Centers for Disease Control and Prevention, "Updated interim recommendations for the use of antiviral medications in the treatment and prevention of influenza for the 2009-2010 season," 2009, http://www.cdc.gov/h1n1flu/ recommendations.htm.
[14] Infectious Diseases Society of America, "Influenza H1N1: frontline questions and expert opinion answers," 2009, http:// www.idsociety.org/Content.aspx?id=15743.

[15] World Health Organization, "Clinical management of human infection with pandemic (H1N1) 2009: revised guidance," 2010, http://www.who.int/csr/resources/publications/ swineflu/clinical_management/en/index.html.

[16] C. H. Hsu, K. C. Hwang, C. L. Chao, S. G. N. Chang, M. S. Ho, and P. Chou, "Can herbal medicine assist against avian flu? Learning from the experience of using supplementary treatment with Chinese medicine on SARS or SARS-like infectious disease in 2003," Journal of Alternative and Complementary Medicine, vol. 12, no. 6, pp. 505-506, 2006. 


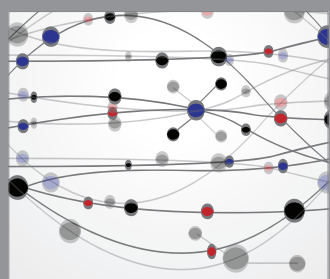

The Scientific World Journal
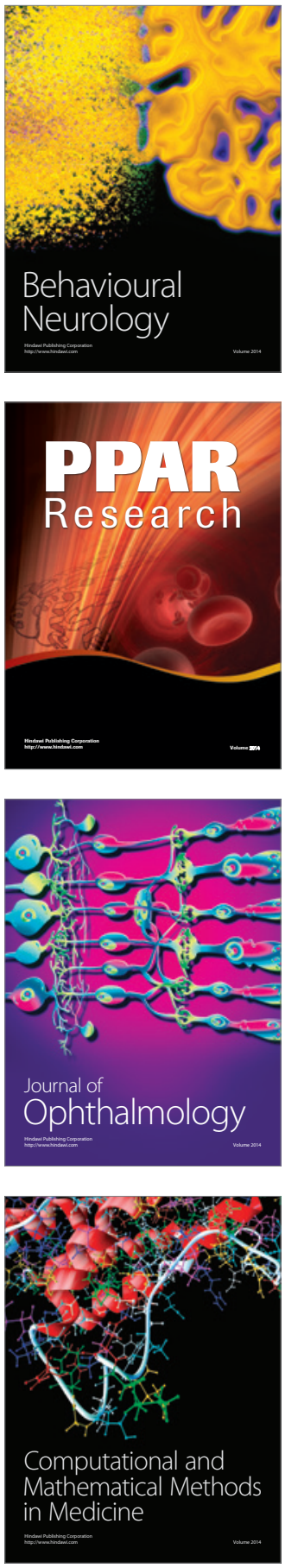

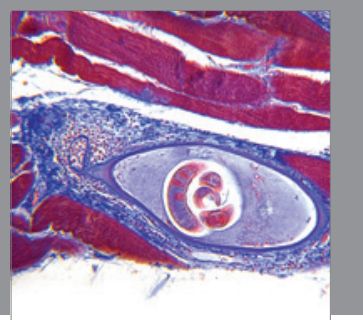

Gastroenterology

Research and Practice
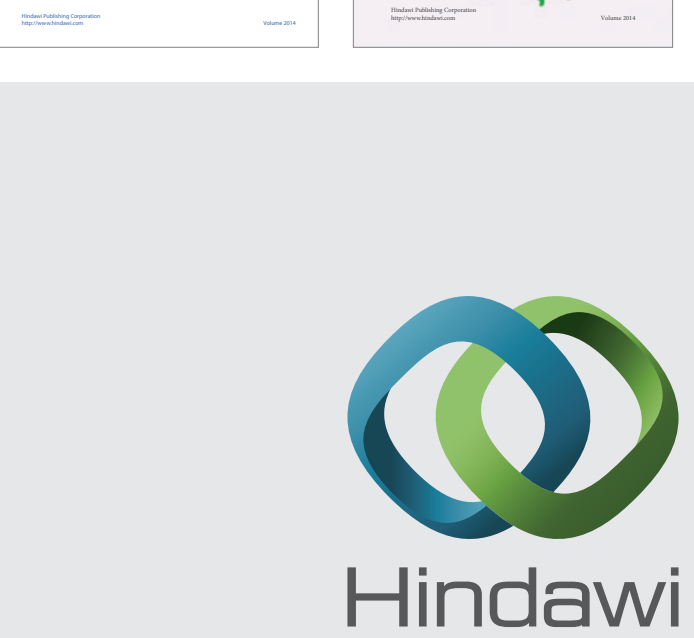

Submit your manuscripts at

http://www.hindawi.com
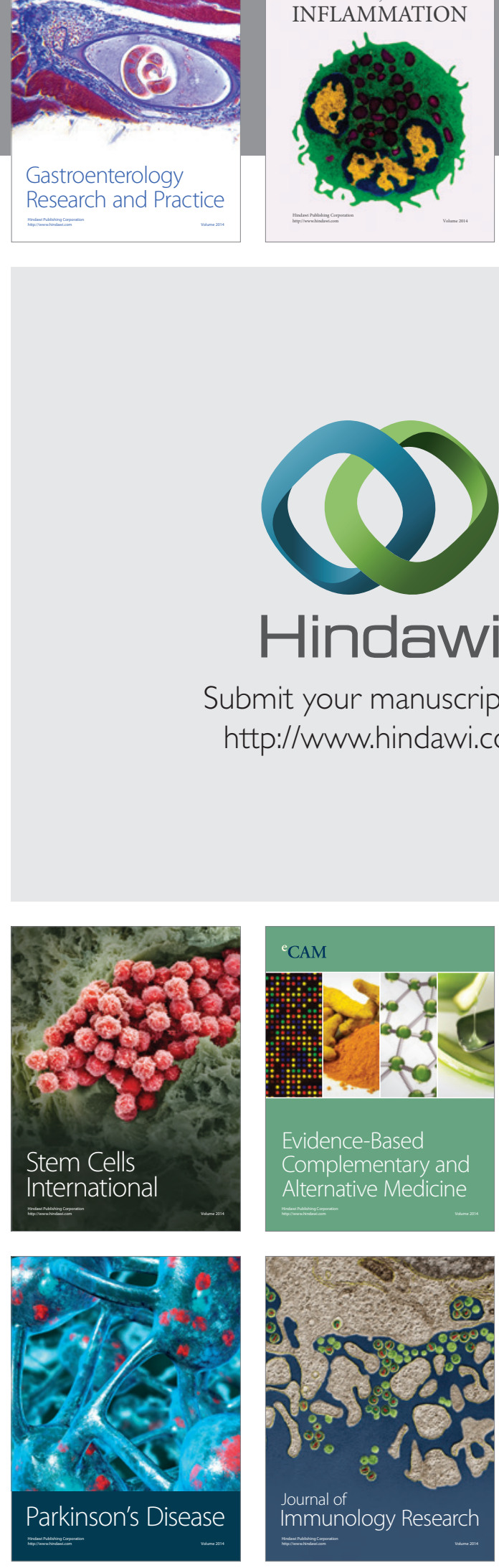

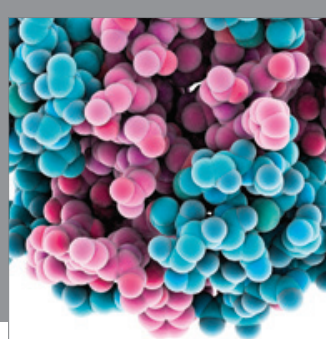

Diabetes Research
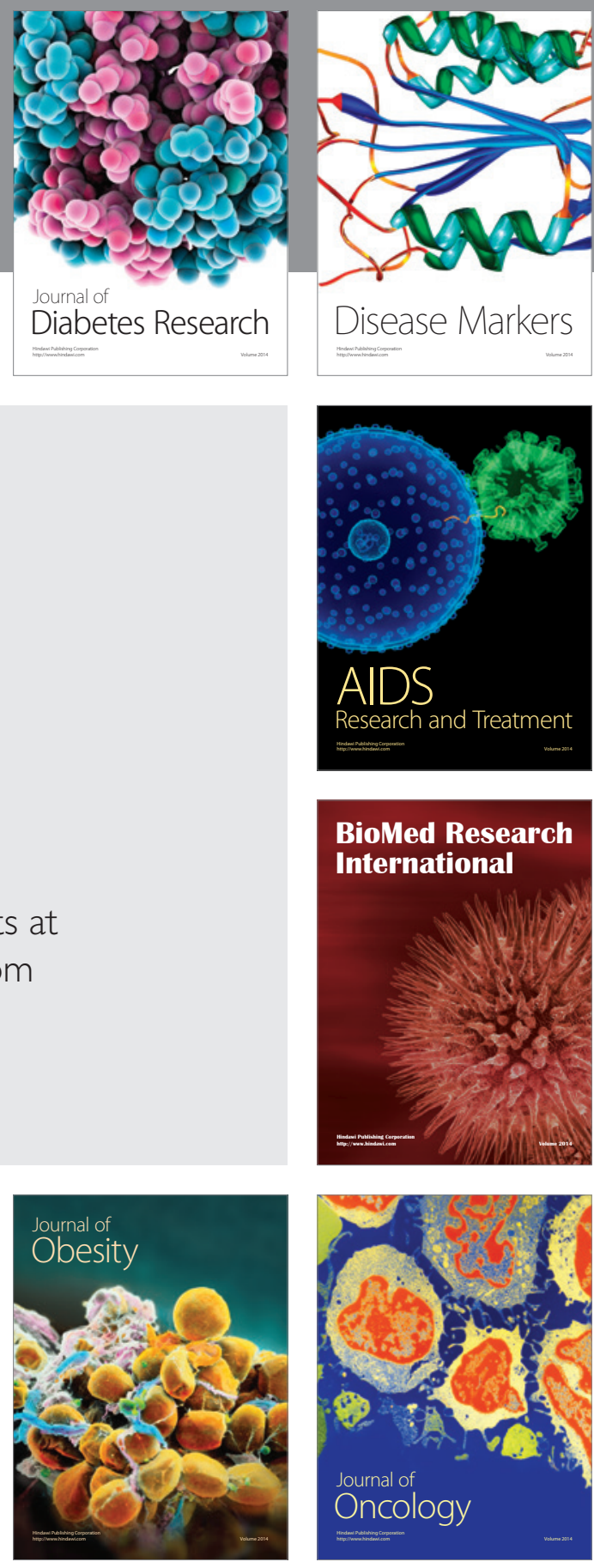

Disease Markers

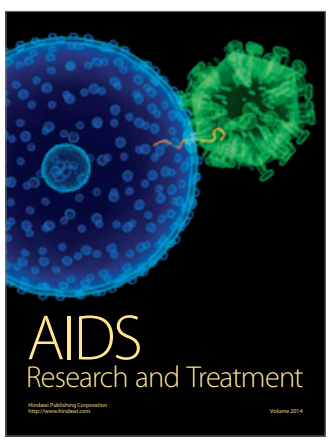

BioMed Research

International
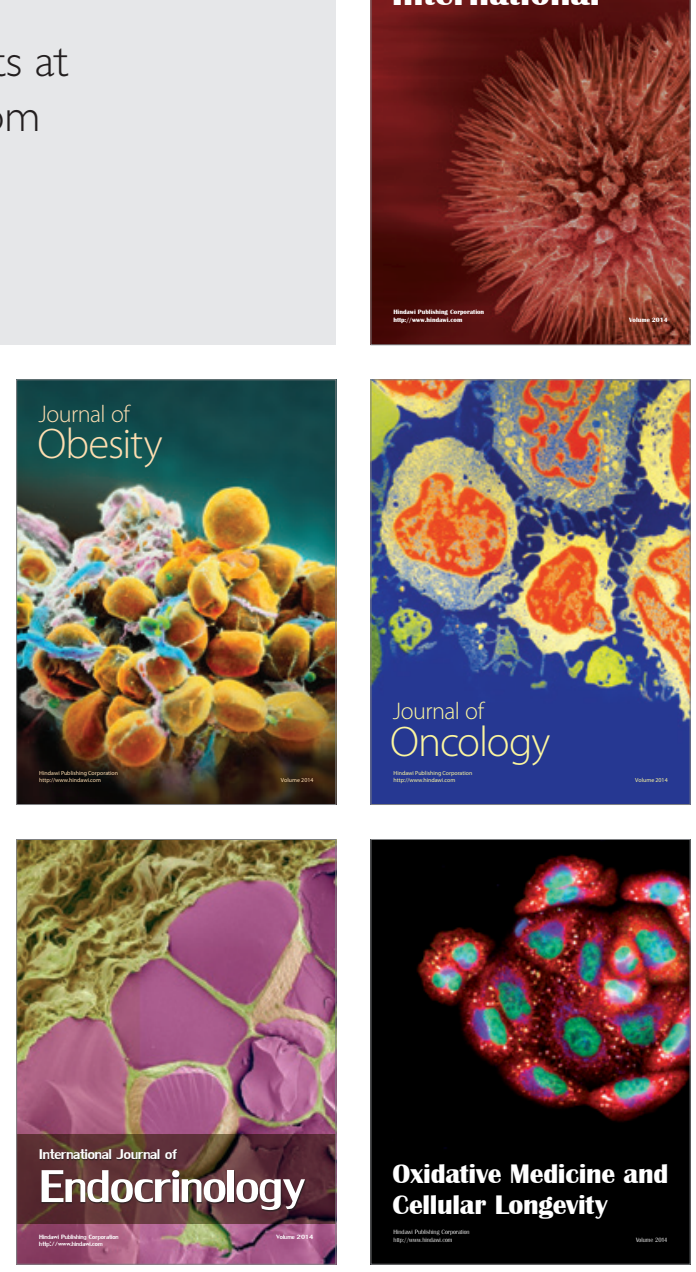